\title{
Image-retrieval system aids cervical-cancer studies
}

Zhiyun Xue, Sameer Antani, L. Rodney Long, and George R. Thoma

A Web-accessible, content-based system for searching a large cervigram database may play an important role in medical education and cervicalcancer research.

Cervical cancer is the second-most common cancer affecting women worldwide. Cervicographic images (cervigrams) taken during gynecological exams (see Figure 1) are important tools for diagnosis and research of the disease. The US National Library of Medicine (NLM), in collaboration with the National Cancer Institute (NCI), is creating a large repository with about 100,000 cervigrams and related text and visual clinical data. The information was collected during two major NCI-funded projects, 'Guanacaste' and 'ALTS' (ASCUS-LSIL Triage Study). ${ }^{1}$ NLM, assisted by gynecological experts, has been developing a multimedia-database tool (MDT) ${ }^{2}$ for text-based searches. Though valuable, it is deficient for retrieving images with particular visual characteristics, such as cervical lesions of specific color, texture, size, or location. This has motivated research and development in content-based, image-retrieval (CBIR) techniques. Complementary to MDT, CBIR searches may become important for accessing the Guanacaste and ALTS data and studying the visual precursors to cervical cancer.

The NLM's CervigramFinder is believed to be the first Web-accessible CBIR software tool for cervigrams. ${ }^{3}$ Oncological gynecologists segmented and labeled the database by medically significant regions that may indicate innocuous or precancerous physiological change. The system is aimed at bridging the semantic gap between the user's understanding of the image content and the machine's representation of its quantitative features.

In most approaches to CBIR, the fundamental search unit is the whole image. This approach is not satisfactory for our application, since physicians interpreting a cervigram must assess visual characteristics such as the 'acetowhite areas' or the 'transformation zone'. To address this problem, CervigramFinder searches for local image characteristics.

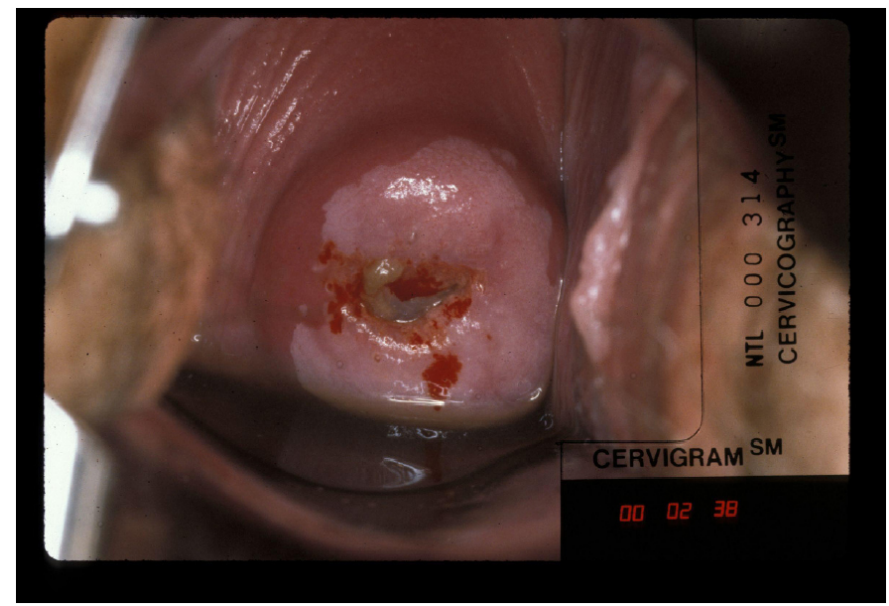

Figure 1. Cervigram.

With the system's graphical user interface (see Figure 2), users may select a query image, draw their own region on it, and specify which visual features (color, texture, size, and location) should be searched. They can refine the query by specifying the relative importance of the features. The system then computes the query region's feature vector (numerical feature representing an object) and compares it with pre-computed feature vectors of database image regions. The resulting regions are ranked by degree of similarity to the query-feature vector. The images containing these regions are displayed with associated patient data.

The CervigramFinder relies on a client/server distributed computer architecture (see Figure 3) that is Web-accessible. The system uses open standards and software, and decouples the user interface from the core indexing and retrieval algorithms. It consists of four modules: a Java applet (program) client with the graphical user interface, a Java servlet (programming language object) gateway for Web and client-server communication, the indexing and retrieval server that extracts features and computes similarity, and the database that contains text and 


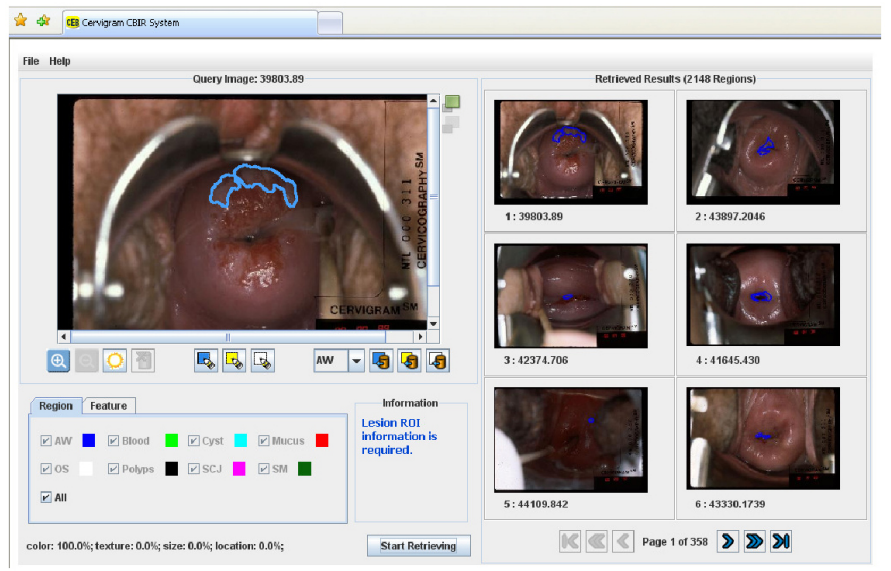

Figure 2. CervigramFinder graphical user interface.
Server Side

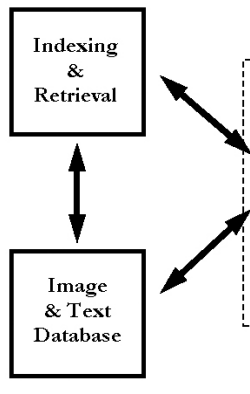

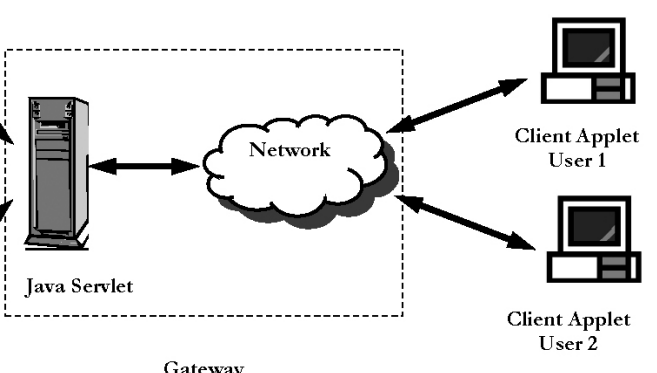

Client Side
Figure 3. CervigramFinder system architecture with a gateway to the Web.

image data from the patient record. CervigramFinder's modular design should allow for user interface enhancements and new algorithms.

Interdisciplinary researchers in engineering and medicine expect CBIR-based technologies to play an important role in the future use of biomedical-image databases for clinical applications and education. Software tools, such as CervigramFinder, represent a significant effort toward developing what may become essential capabilities of biomedical image-database systems. If CervigramFinder can contribute to medical education and research into cervical cancer, large numbers of women who suffer from the disease could benefit.

The authors acknowledge the medical expertise of Mark Schiffman, $M D$, of NCI, and Jose Jeronimo, MD, (formerly with NCI) of the Program for Appropriate Technology in Healthcare. This research was supported by the Intramural Research Program of the National Institutes of Health (NIH), the NLM, and the Lister Hill National Center for Biomedical Communications.

\section{Author Information}

\section{Zhiyun Xue}

Lister Hill National Center for Biomedical Communications US National Library of Medicine (NLM)

Bethesda, MD

Lockheed Martin MSD Inc.

Fairfax, VA

Zhiyun Xue works on image retrieval. She received her $\mathrm{PhD}$ in electrical engineering from Lehigh University, and her MS and BS in electrical engineering from Tsinghua University, China. Her research interests include medical-image analysis, computer vision, and machine learning.

\section{Sameer Antani, L. Rodney Long, and George R. Thoma}

Lister Hill National Center for Biomedical Communications US National Library of Medicine (NLM)

Bethesda, MD

Sameer Antani, staff scientist at the NLM and the National Institutes of Health (NIH), studies multimodal (image and text) biomedical informatics and next-generation, multimedia-rich scientific publications. He is a member of the IEEE, IEEE Computer Society, and SPIE.

L. Rodney Long is an electronics engineer for the NLM's Communications Engineering Branch. He worked for 14 years in industry as a software developer and systems engineer. His research interests are telecommunications, image processing, and scientific/biomedical databases.

George R. Thoma, an NLM branch chief, directs research and development programs in document-image analysis, biomedical imaging, and related areas. He earned a BS from Swarthmore College, and an MS and PhD from the University of Pennsylvania, all in electrical engineering. He is a SPIE Fellow.

\section{References}

1. R. Herrero, M. H. Schiffman, C. Bratti, et al., Design and methods of a populationbased natural history study of cervical neoplasia in a rural province of Costa Rica: the Guanacaste Project, Rev. Panam Salud Publica 1 (5), pp. 362-375, 1997. doi:10.1590/S1020-49891997000500005

2. L. R. Long, S. Antani, and G. R. Thoma, Image informatics at a national research center, Comput. Med. Imaging Graphics 29, pp. 171-193, 2005. doi: 10.1016/ j.compmedimag.2004.09.015

3. Z. Xue, S. Antani, L. R. Long, J. Jeronimo, and G. Thoma, A Web-accessible, content-based cervicographic image-retrieval system, Proc. SPIE 6919, pp. 691907-1-9, 2008 . 